\title{
Coculturing NSCs with Melanocyte Increased its Dopamine and Neural Factor Secretion
}

\author{
Ashok Chakraborty ${ }^{*}$ and Anil Diwan ${ }^{2}$ \\ ${ }^{1}$ Chief Scientist, Allexcel, Inc., USA \\ ${ }^{2}$ Chief Executive Officer, Allexcel, Inc., USA \\ *Corresponding Author: Ashok Chakraborty, Chief Scientist, Allexcel, Inc., USA.
}

Received: December 11, 2020

Published: April 30, 2021

(C) All rights are reserved by Ashok

Chakraborty and Anil Diwan.

\begin{abstract}
Background: Neural stem cells (NSCs) since being a multi-potent neural crest originated cells, are able for self-replication, and differentiation to astrocytes, oligodendrocytes, or neurons. Therefore, it is expected that autologous transplantation of hNSCs to CNS, can be considered as a therapeutic approach for Parkinson's disease (PD). However, growth of human neural stem cells (hNSCs) is very slow, and they senesce after few passages, therefore enough amount of neural cells is very difficult to obtain to treat a number of patients.

Aim: The growth potential and survival length of the neural stem cells have to improved to make it practically feasible to use as a cell therapeutic regiment for PD. At the present time, cell co-cultures have been extensively used in cell functional research. We will study here whether co-culturing NSCs with another neural crest originated cells, Melanocytes (MCs), can increase the proliferation rate and Dopamine synthesis capacity of NSCs.

Methods: We compared the growth potential, synthesis of DOPAmine (DA), Brain-Derived Neurotropic Factor (BDNF) and Glial cellDerived Neurotropic Factors (GDNF) by NSCs cultured alone and in co-culture system with MCs.

Results: hNSC's doubling time increases by 2-3 times in co-culture condition. Survival length of hNSCs in co-culture system is beyond 25 passages, whereas senescence occurred after only 5-6 passages when cultured alone. DA production as well as BDNF and GDNF production by hNSCs is much more in co-culture system compared to hNSCs by itself, only.

Conclusion: A possible modification of hNSCs is possible by close association with melanocytes. A further study is required how these changes can be made beyond transient and subtle type.

Keywords: Human Neural Stem Cells (hNSCs); DOPAmine; Dopaminergic Neurons; BDNF; GDNF; Parkinson's Disease; Melanocytes; Co-Culture System
\end{abstract}

\section{Introduction}

Parkinson's disease is one kind of neuro-degenerative disease develops due to the loss of dopaminergic neurons in the substantia nigra (SN) [1,2]. Human neural stem cells (hNSCs) are able to differentiate into mature neurons, and can secrete a number of neurotropic factors, (BDNF, GDNF), that helps regeneration of damaged neurons, also $[3,4]$.
Furthermore, NSCs are also known to be the subject to exogenous regulation of the micro-environment including various structural support, adjacent cells and extracellular matrices, that promotes proliferation and differentiation of NSCs [5-7].

Interplay between neural stem cells and glioblastoma induces the rate of proliferation, differentiation of NSCs to neurons [8]. 
Transplantation of NSCs along with astrocytes into the ischemic striatum of middle cerebral artery occlusion (MCAO) rat model improves the survival and neuronal differentiation of transplanted NSCs than when transplanted alone [9]. Sertoli cells (SCs) derived from the testis, provide growth factors for the development of germinal and neural stem cell. Co-culturing of primary mouse Sertoli cells with neural stem cells (NSCs) from mouse resulted highest neural outgrowths and neurospheres, and the increased amount of secretory proteins [10].

GDNF (glial cell-derived neurotrophic factor) and BDNF (brainderived neurotrophic factor) are known to have positive role in growth, survival length, and differentiation of developing neurons [11-16]. These factors can also inhibit the progressive loss of DAergic neurons in vivo (PD animal model), and also promotes the survival of dopaminergic neurons in culture [11,17].

Here we show that hNSCs, in vitro, can produce an increased amount of BDNF, GDNF and DOPAmine (DA) in co-culture system with melanocytes (MCs) compared to when cultured alone.

\section{Materials and Methods}

Culture of human neural stem cells (hNSCs)

Adult human neural stem cells (H9) derived from female origin were procured from ThermoFisher (Rockford, IL, USA). The growth and maintenance of were done as described previously [19]. In brief, 6-well tissue culture plates were pre-coated with cell-matrix gel (ATCC, Manassas, VA), and cells were cultured in them with complete growth medium at $37^{\circ} \mathrm{C}$ in a $5 \% \mathrm{CO}_{2}$ humidified incubation chamber.

Accutase, a cell dissociation reagent, (ATCC, Manassas, VA), were used for subculturing and passaging of the hNSC spheres into a single cells.

\section{Proliferation assay}

Cell-Titer-Glo Luminescent Assay kit (Promega, Madison, WI) was used to detected the viability as well as the rate of proliferation of cells, as described in the manufacturer's protocol. In brief, for in vitro growth assay, $1 \times 10^{3}$ cells were plated in each well of a 96-well tissue culture plate. Next day, '0 day', followed by every alternate day cell growth was measured using the Cell-Glo reagent until for 6 days. Luminescence readings were taken using the BioTek plate reader [18]. Results were expressed as percentage of vehicle-control cells from 3 well readings. The experiments were repeated three times with similar results.

\section{ELISA for tyrosine hydroxylase}

Tyrosine hydroxylase enzyme-link immunosorbent assay (THELISA) was performed as described previously [19]. Total protein extraction from cells were done by using RIPA lysis buffer. Protein concentrations were calculated using the Bradford reagent (SigmaAldrich), and BSA as a standard.

\section{DOPAmine assay by ELISA}

Dopamine production by cells was measured by ELISA as described in the manufacturer's protocol (Eagle Bioscience, Nashua, $\mathrm{NH}$; Cat\# DOP31-K01). Every sample for dopamine assay and standard dopamine solutions were performed in triplicate. Absorbance readings were taken at $450 \mathrm{~nm}$ set to $450 \mathrm{~nm}$ with a reference wavelength set between 620 and $650 \mathrm{~nm}$. Date were taken in triplicate for each sample.

Assay of BDNF and GDNF protein concentration by ELISA

ELISA was performed to determine the secretion of BDNF and GDNF by hNSCs at different days and in different experimental conditions [20]. First, the Elisa plates were pre-coated with antibody against human BDNF and/or GDNF on a 96-well plate. The Standards and the test samples were added sequentially to that wells. Samples were then incubated for 120 minutes at $37^{\circ} \mathrm{C}$. After the incubation period, the plates were washed with the washing buffer (supplied with the kit) three times, and allowed to soak for 1 minute in a filter paper each time. The biotinylated anti-human BDNF or GDNF antibody ( $50 \mu \mathrm{L} /$ well) was added to each appropriate wells containing samples. The plates were incubated at $37^{\circ} \mathrm{C}$ for 60 minutes, followed by washing 4 times with the wash buffer. Subsequently, HRP-conjugated streptavidin is pipetted to the wells and incubated for 45 minutes at $37^{\circ} \mathrm{C}$ in the dark with gentle shaking. One-step substrate reagent (TMB) was added to each well followed by incubation for 30 mints. at room temperature in the 
dark with gentle shaking. The reaction was stopped by the addition of 3,3', 5,5'-tetramethyl benzidine stopping solution (50 $\mu \mathrm{L} /$ well), and the color formation in each well were measured at 450 nm immediately. The standard curve was generated by plotting the absorbance on the vertical (Y) axis corresponding to the concentration on the horizontal $(\mathrm{X})$ axis.

\section{Protein assay}

Protein amount was measured by Bio-Rad Protein Assay Kit (Bio-Rad, Richmond, CA) with bovine serum albumin as a standard.

\section{Cell counting and viability detection}

Cell count and viability measurements were done using standard procedure, like staining the cells with Trypan Blue and then counting the number of viable cells with the Countess ${ }^{\mathrm{TM}}$ automated cell counter (Invitrogen).

\section{Statistical analysis}

The $t$-test and one-way analysis of variance were used for statistically analyzing the data. All values are expressed as the Average \pm SD. $P$ values less than 0.05 were considered as significant. Results are representative of at least three experiments done in triplicate.

\section{Results}

- Increased Growth and survival length of hNSCs in coculture condition: hNSCs were grown in media alone and in co-culture condition with hMCs, as described in the Materials and Method section. Photographs are taken by Axiovert-100 Microscope (Zeiss, German) in different indicated days with magnification of objectives at $20 \mathrm{X}$.

- Increased TH expression and DA production by hNSCs in co-culture system: hNSCs express Tyrosine hydroxylase enzyme which converts tyrosine to Dopamine (DA). Dopamine production and expression of Tyrosine hydroxylase by hNSC were measured using the respective ELISA kits as described in the Materials and Methods. Values were normalized per $\mathrm{mL}$ of the cell extracts containing same number of cells. The results were placed from a representative experiment done in triplicate.

Results show that the expression level of $\mathrm{TH}$ and Dopamine production by hNSCs are induced in co-culture condition. However,
MCLts, the effector cells, do not show any expression of TH or produces dopamine at any detectable level.

- Increased Production of BDNF/ GDNF by hNSCs in Coculture (Table 1): The levels of BDNF or GDNF produced by hNSCs when cultured alone or in co-culture condition with nhMCs, were measured by ELISA. The values for BDNF were expressed as ng/ $10^{5}$ Cells, and GDNF as pg/ $10^{5}$ Cells. Each experimental data point are the Average \pm SD of three data points from each condition, $\mathrm{P}<0.05$. The experiment was repeated three times with similar results.

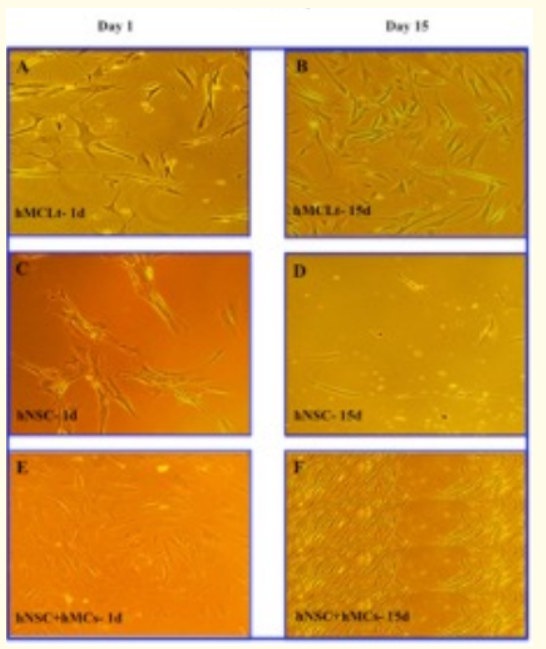

Figure 1: Effect on morphology and growth of hNSCs co-culturing with melanocytes.

MCLts (Normal Human melanocytes from male origin) and hNSCs (Normal human neural stem cells from female origin) were grown in their preferred media and in a gel-coated tissue culture plates as described in the Materials and Method section. (A) Morphology: Co-culture group (panel E, and F), shows neuron cell bodies were translucent with a slender shape and multiple processes, typical of neurons. (B) Growth: Neural stem cells group, a smaller amount of neuron cells were present (panel E), and the state of neurons were not as healthy as in the co-culture group (panel F). Scale bar, $100 \mu \mathrm{m}$. 


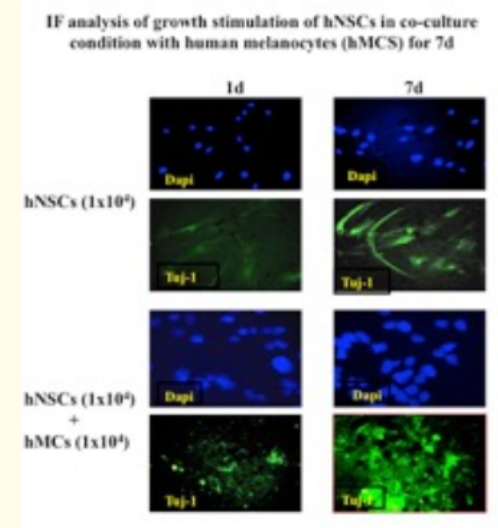

Figure 2: Immunofluorescence (IF) analysis of hNSCs in co-culture condition with human melanocytes (hMCs) for $7 \mathrm{~d}$.

Immunofluorescence study of hNSCs cells were done using some NSC-specific antibody (Tuj-1, green) in different culture condition for 7 days. DAPI blue stains were done for nucleus staining, as described in the Materials and Method section. Photographs were taken by Axiovert-Fluorescence Microscope (Zeiss, German). The magnification of objectives was 20X and total magnification were 200X. It is obvious from the figure that NSCs grows more in co-culture system than when cultured alone over the same period of time. DAPI: 4',6-diamidino-2-phenylindole.

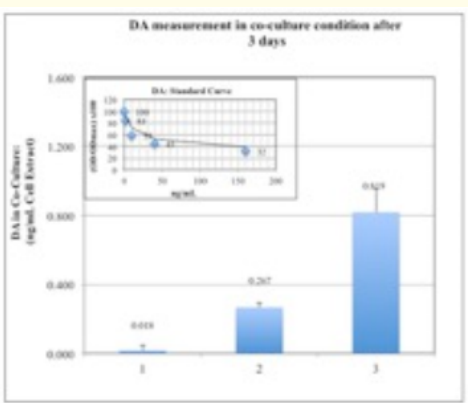

Figure 3: Dopamine (DA) measurements in Co-culture condition after 3days.

Biochemical analysis of Dopamine release by MCLts, hNSCs and in co-culture conditions were done in triplicate. Unlike MCLts, human neural cells (hNSCs) can synthesize and release Dopamine in the medium, and further increase in synthesis were observed over the same period of time. Details of assay are described in the Materials and Methods section. Values are calculated from the standard Curve (inset), and are expressed as $\mathrm{ng} / \mathrm{mL}$ cell extract). The data are from a representative experiment done in triplicate. Percentage of error are all within $10 \%$. 


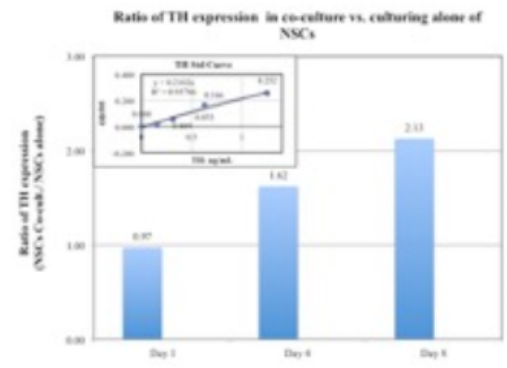

Figure 4: Ratio of Tyrosine Hydroxylase (TH) expression in co-culture vs. culturing alone of NSCs.

To confirm quantitatively the dopaminergic differentiated hNSCs, the tyrosine hydroxylase enzyme-link immunosorbent assay (THELISA) was performed (Donald et. al. 2013). Total protein extracted from cells, undifferentiated hNSCs and in presence of differentiated media, were prepared using RIPA lysis buffer. Protein concentrations were determined by the Bradford method (Sigma-Aldrich), using BSA as the standard (Thermo Scientific). After verifying the final protein concentrations, the same concentration of protein was used in all the samples. All samples were used to measure the levels of TH, according to the Kit manufacturer's protocol (USCNK Life Science Inc.,

Wuhan, China).
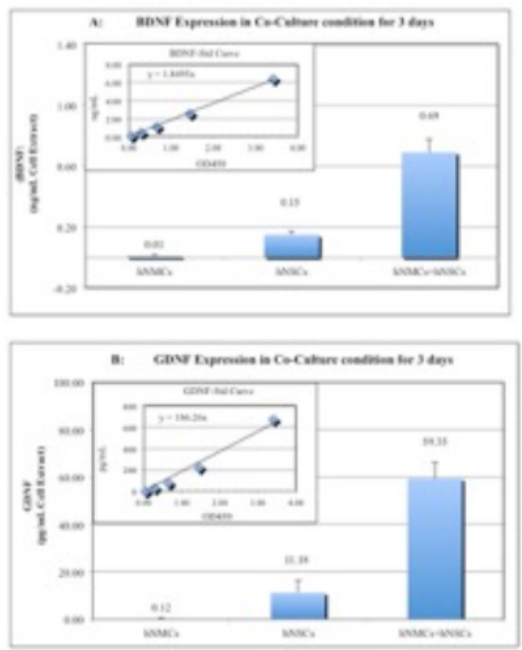

Figure 5: BDNF/GDNF Expression by hNSCs in Co-Culture condition with MCs for 3 days.

The levels of BDNF or GDNF production by hNSCs alone in culture and in co-culture were compared by measuring those proteins in cell lysates by ELISA. BDNF level was expressed as ng/105 Cells, while GDNF was expressed as pg/mL cell extracts. The experimental data are Mean \pm SD from three separate experiments done in duplicate. $\mathrm{P}<0.05$. Inset is the Standard Curve of BDNF (Panel A) and GDNF (Panel B). 


\section{Discussion and Conclusion}

Co-culture system, now-a-days, have been extensively studied in understanding of the cellular behavior. The effector cells can induce the other cell's proliferation, viability and also differentiation into other types of cells. Endothelial cells (ECs) have been found to stimulate the proliferation and differentiation of NSCs, probably by via VEGF, wnt, Notch, and Pten signaling pathways. It was reported before that the expression of the Wnt signaling genes (Wnt3a and $\beta$-catenin), the Notch signaling genes (notch2, numb, Hes1, and Psen1), and the Pten signaling pathway-genes (Pten, Akt1, and PIP3), and increased significantly in an in vitro co-culture conditions [5-7].

Microglia, collected from ischemic or exo-cytotoxic injured brain, can stimulate NSCs in culture to release mitogenic factors, promotes proliferation of NSCs, it's differentiation to neurons and formation of oligodendrocytes [22]. Co-transplantation of both astrocytes and NSCs into the ischemic striatum of the MCAO (middle cerebral artery occlusion) rat model, resulted higher survival, proliferation, and differentiation to neuron of the transplanted NSCs [9].

Bone marrow-derived mesenchymal stem cells (BM-MSCs) are known for its wide use in tissue engineering. Stimulation of proliferation and differentiation of NSCs have been found when cocultured with BM-MSCs. In addition, BM-MSCs can also protect NSCs from neutrotoxic effect of 6-hydroxy dopamine [23]. All those effects were due to the upregulation of chemokine ligand-2 (CCL2) released from BM-MSCs [24]. Taken together, the mechanism of neurogenesis stimulated by BM-MSCs is interesting. The similar behavior also have been noticed with Olfactory ensheathing cell (OEC) in stimulating the proliferation, neuronal differentiation of NSCs in co-culture system [25].

Biologically active neurotropins, microRNAs, many growth factors, are involved in the endogenous regulation of proliferation and differentiation of hNSCs. Moreover, microenvironment, like adjacent cells and extracellular can also exert exogenous regulation of NSCs for its proliferation and differentiation to neurons [26]. Therefore, it is clear that the regulation of NSCs behavior is closely related to the cell signaling pathway and the expression of the related genes. Brain-derived neurotrophic factor (BDNF) has $54 \%$ sequence homology to NGF (nerve growth factor) and serves for survival and growth of motor neurons, and expression of cholinergic genes [27-29]. Glial cell line-derived neurotrophic factor (GDNF) are closely linked with the transforming growth factor family, and promotes motor and sensory neuron survival [30-33]. These neurotropic factors also play an important roles in synaptic plasticity, learning and memory [11]. Further, GDNF stimulates the expansion of nestin-positive neural precursors and increases the diameter of neurosphere. However, these events are related to the up-regulation of Bcl-2 and down-regulation of caspase-3, a marker of anti-apoptotic response [33].

Roussa and Krieglstein (2004) have shown that GDNF though does not increase TH expression in neurospheres but increases the expression of several transcription factors (Nurr1 and Pitx3) involved in dopaminergic phenotype [34]. Later on, Nurr1 was shown to induce the GDNF co-receptor, c-Ret. Therefore, Nurr1expressing cells when exposed to GDNF becomes more responsive to GDNF, and up-regulates the Pitx3 expression and ultimately the dopaminergic phenotype [35]. In parallel, an increase in Dopamine transporter (DAT) activity was found with GDNF [36]. Inhibitors of ERK1/2 can prevent GDNF signaling associated with dopaminergic phenotype [37]. All TH+ neurons express Dlk1, and GDNF treatment can significantly increase $D l k 1$ expression and dopaminergic phenotype, as well, in human mesencephalic cells [38]. Thus, GDNF offers not only the neurons-specific phenotypes but also increases the survival length of neurons. In neurons, the outgrowth of the dendrites and electrophysiological maturation occurs simultaneously, that stimulates potassium depolarization and DA release. In addition, there are various examples where GDNF or BDNF treatment has shown to increase DA release significantly, like in mesencephalic cultures [39] or in postnatal cultures [40]. GDNF exhibits potential therapeutic importance for several pathological conditions, such as Parkinson's disease (PD) or amyotrophic lateral sclerosis (ALS) [41-43]. The understanding of broad action mechanism of GDNF, as well as BDNF may provide an opportunity to accelerate and/or improve therapies for neural defects.

In conclusion, the current study confirmed that co-culturing of NSCs with MCs in vitro causes a positive changes in cell growth, sur- 
vival length of hNSCs, and also increased it's capability to release DA and neural supportive factors, BDNF/GDNF. The broad understanding of intercellular cross-reactivity and the involvement of the trophic molecules will shed lights for the improvement of therapies for neuro-degenerative diseases, like Parkinson's Disease.

\section{Acknowledgement}

This research is supported by internal grant from AllExcel, Inc.

\section{Conflict of Interest}

The authors report no conflicts of interest.

\section{Bibliography}

1. Alexander GE. “Biology of Parkinson's Disease: pathogenesis and pathophysiology of a multisystem neurodegenerative disorder". Dialogues in Clinical Neuroscience 6.3 (2004): 259-280.

2. Zeng X-S., et al. "Cellular and Molecular Basis of Neurodegeneration in Parkinson Disease". Frontiers in Aging Neuroscience 10 (2018): 109.

3. Rodriguez AM., et al. "Transplantation of a multipotent cell population from human adipose tissue induces dystrophin expression in the immunocompetent mdx mouse". Journal of Experimental Medicine 201 (2005): 1397-1405.

4. Mosahebi A., et al. "Retroviral labeling of Schwann cells: In vitro characterization and in vivo transplantation to improve peripheral nerve regeneration". Glia 34 (2001): 8-17.

5. Shen Q., et al. "Endothelial cells stimulate self-renewal and expand neurogenesis of neural stem cells". Science 304. 5675 (2004): 1338-1340.

6. Sun J., et al. "Endothelial cells promote neural stem cell proliferation and differentiation associated with VEGF activated Notch and Pten signaling". Developmental Dynamics 239.9 (2010): 2345-2353.

7. Du YB., et al. "Wnt3a is critical for endothelial progenitor cellmediated neural stem cell proliferation and differentiation". Molecular Medicine Reports 14 (2016): 2473-2482.
8. Wang G., et al. "Synergistic effect of neural stem cells and olfactory ensheathing cells on repair of adult rat spinal cord injury". Cell Transplantation 19.10 (2010): 1325-1337.

9. Luo L., et al. "Niche astrocytes promote the survival, proliferation and neuronal differentiation of co-transplanted neural stem cells following ischemic stroke in rats". Experimental and Therapeutic Medicine 13.2 (2017): 645-650.

10. Shi B., et al. "The enhancement of neural stem cell survival and growth by co-culturing with expanded Sertoli cells in vitro". Biotechnology Progress 28.1 (2012): 196-205.

11. Lin LF., et al. "GDNF: a glial cell line-derived neurotrophic factor for midbrain dopaminergic neurons". Science 1260 (1993): 1130-1132.

12. Hyman C., et al. "BDNF is a neurotrophic factor for dopaminergic neurons of the substantia nigra". Nature 350.6315 (1991): 230-232.

13. Klein RL., et al. "Prevention of 6-hydroxydopamine-induced rotational behavior by BDNF somatic gene transfer". Brain Research 847 (1999): 314-320.

14. Bespalov MM and Saarma M. "GDNF family receptor complexes are emerging drug targets". Trends in Pharmacological Sciences 28 (2007): 68-74.

15. Chao MV. "Neurotrophins and their receptors: a convergence point for many signaling pathways". Nature Reviews Neuroscience 4 (2003): 299-309.

16. Chao MV., et al. "Neurotrophin signalling in health and disease". Clinical Science (Lond) 110 (2006): 167-173.

17. Akerud P., et al. "Neuroprotection through delivery of glial cell line-derived neurotrophic factor by neural stem cells in a mouse model of Parkinson's disease". Journal of Neuroscience 21 (2001): 8108-8118.

18. Crouch SP., et al. "The use of ATP bioluminescence as a measure of cell proliferation and cytotoxicity". Journal of Immunological Methods 160 (1993): 81-88. 
19. Chakraborty A and Diwan A. "Selection of Cells for Parkinson's Disease Cell-Therapy". International Journal of Stem cell Research and Therapy 6 (2019): 063.

20. Erickson JT., et al. "Brain-Derived Neurotrophic Factor and Glial Cell Line-Derived Neurotrophic Factor Are Required Simultaneously for Survival of Dopaminergic Primary Sensory Neurons In Vivo". The Journal of Neuroscience 21.2 (2001): 581-589.

21. Chakraborty A and Diwan A. "Autocrine and paracrine stimulation of dopamine secretion by human neural stem cells: Role BDNF and GDNF". Neurology and Neuroscience Reports 3 (2020): 1-6.

22. Deierborg T., et al. "Brain injury activates microglia that induce neural stem cell proliferation ex vivo and promote differentiation of neurosphere-derived cells into neurons and oligodendrocytes". Neuroscience 171.4 (2010): 1386-1396.

23. Habisch HJ., et al. "Neuroectodermally converted human mesenchymal stromal cells provide cytoprotective effects on neural stem cells and inhibit their glial differentiation". Cytotherapy 12 (2010): 491-504.

24. Lee H., et al. "Bone-marrow-derived mesenchymal stem cells promote proliferation and neuronal differentiation of Niemann-Pick type $\mathrm{C}$ mouse neural stem cells by upregulation and secretion of CCL2". Human Gene Therapy 24.7 (2013): 655-669.

25. Wang G., et al. "Synergistic effect of neural stem cells and olfactory en-sheathing cells on repair of adult rat spinal cord injury". Cell Transplantation 19.10 (2010): 1325-1337.

26. Ramon-Cueto A and Valverde F. "Olfactory bulb ensheathing glia: a unique cell type with axonal growth-promoting properties". Glia 14.3 (1995): 163-173.

27. Leibrock J., et al. "Molecular cloning and expression of brainderived neurotrophic factor". Nature 341 (1989): 149-152.

28. Squinto SP., et al. "trkB encodes a functional receptor for brainderived neurotrophic factor and neurotrophin-3 but not nerve growth factor". Cell 65 (1991): 885-893.
29. An YH., et al. "Effect of rat Schwann cell secretion on proliferation and differentiation of human neural stem cells". Biomedical and Environmental Sciences 16 (2003): 90-94.

30. Krieglstein K., et al. "Glial cell line-derived neurotrophic factor requires transforming growth factor-beta for exerting its full neurotrophic potential on peripheral and CNS neurons". Journal of Neuroscience 18.23 (1998): 9822-9834.

31. Airaksinen M and Saarma M. "The GDNF family: Signalling, biological functions and therapeutic value". Nature Reviews Neuroscience 3 (2002): 383-394.

32. Saarma M. "GDNF - a stranger in the TGF-beta superfamily?" European Journal of Biochemistry 267.24 (2000): 6968-6971.

33. Lei Z., et al. "Signaling of glial cell line-derived neurotrophic factor and its receptor GFR $\alpha 1$ induce Nurr1 and Pitx3 to promote survival of grafted midbrain-derived neural stem cells in a rat model of Parkinson disease". Journal of Neuropathology and Experimental Neurology70 (2011): 736-747.

34. Roussa E and Krieglstein K. "Induction and specification of midbrain dopaminergic cells: focus on SHH, FGF8 and TGFbeta". Cell Tissue Research 318.1 (2004): 23-33.

35. Peng C., et al. "Pitx3 is a critical mediator of GDNF-induced BDNF expression in nigrostriatal dopaminergic neurons". Journal of Neuroscience 31 (2011): 12802-12815.

36. Zhu SY., et al. "Identification of a Vav2-dependent mechanism for GDNF/Ret control of mesolimbic DAT trafficking". Nature Neuroscience 18 (2015): 1084-1093.

37. Consales C., et al. "GDNF signaling in embryonic midbrain neurons in vitro". Brain Research 1159 (2007): 28-39.

38. Christophersen NS., et al. "Midbrain expression of Delta-like 1 homologue is regulated by GDNF and is associated with dopaminergic differentiation". Experimental Neurology 204 (2007): 791-801.

39. Feng L., et al. "Differential effects of GDNF and BDNF on cultured ventral mesencephalic neurons". Molecular Brain Research 66 (1999): 62-70. 
40. Pothos EN., et al. "Presynaptic recording of quanta from midbrain dopamine neurons and modulation of the quantal size". Journal of Neuroscience 18 (1998): 4106-4118.

41. Richardson RM., et al. "Interventional MRI-guided putaminal delivery of AAV2-GDNF for a planned clinical trial in Parkinson's disease". Molecular Therapy 19 (2011): 1048-1057.

42. Thomsen GM., et al. "The past, present and future of stem cell clinical trials for ALS". Experimental Neurology 262 (2014): 127-137.

43. Rolan PE., et al. "First-in-human, double-blind, placebo-controlled, randomized, dose-escalation study of BG00010, a glial cell line-derived neurotrophic factor family member, in subjects with unilateral sciatica". PLoS One 10 (2015): e0125034.

\section{Assets from publication with us}

- Prompt Acknowledgement after receiving the article

- Thorough Double blinded peer review

- Rapid Publication

- Issue of Publication Certificate

- High visibility of your Published work

Website: www.actascientific.com/

Submit Article: www.actascientific.com/submission.php

Email us: editor@actascientific.com

Contact us: +919182824667 